\title{
CREENCIAS ACTITUDINALES ENTRE HOMBRES Y MUJERES DE LA CIU- DAD Y EL ESTADO DE MÉXICO HACIA LA VIOLENCIA
}

\author{
Leonel Romero Uribe, Luis Fernando Cruz Maya
}

Universidad Nacional Autónoma de México

\begin{abstract}
Resumen
En las últimas décadas en México se ha ido incrementado el fenómeno de la violencia y la agresión, ya que según AFP (2017) durante el año 2016 México fue el segundo país más violento a causa de las muertes provocadas por el narcotráfico. Por lo anterior se considera que las creencias y actitudes hacia este fenómeno presentes en la población son factores asociados a su incremento. Por ello el propósito de la presente investigación es medir y contrastar las creencias actitudinales de hombres y mujeres de la Ciudad y el Estado de México hacia la violencia. Para ello se hizo una distinción entre los conceptos violencia y agresión, posteriormente se elaboró un instrumento tipo escala Likert con 55 reactivos para evaluar las creencias actitudinales hacia la violencia interpersonal, autoinfligida y colectiva. Se utilizó un estudio descriptivo transversal con diseño ex post facto, con una muestra de 294 participantes. Los resultados, tras aplicarse un análisis factorial, mostraron que existen diferencias significativas en las creencias actitudinales de hombres y mujeres con respecto a la violencia. Además, se observaron correlaciones entre diversos componentes de violencia, esto supone que las diferentes clasificaciones de violencia interactúan para construir un concepto amplio de dicho fenómeno.
\end{abstract}

Palabras clave: Creencias, Actitudes, Violencia, Agresión.

\begin{abstract}
In the last decades in Mexico, the phenomenon of violence and aggression has increased, since according to AFP (2017), in 2016 Mexico was the second most violent country due to the deaths caused by drug trafficking. Therefore, it is considered that the beliefs and attitudes towards this phenomenon present in the population are factors associated with its increase. Therefore, the purpose of this research is to measure and contrast the attitudinal beliefs of men and women in Mexico City and the State towards violence. To do so, a distinction was made between the concepts of violence and aggression, and a Likert scale instrument with 55 items was subsequently developed to assess attitudinal beliefs towards interpersonal, self-inflicted and collective violence. A descriptive crosssectional study with ex post facto design was used, with a sample of 294 participants. The results, after applying a factor analysis, showed that there are significant differences in the attitudinal beliefs of men and women regarding violence. In addition, correlations were observed between various components of violence, this assumes that the different classifications of violence interact to build a broad concept of said phenomenon.
\end{abstract}

Keywords: Beliefs, Attitudes, Violence, Aggression.

\section{Introducción}

De acuerdo con el Instituto Nacional de Estadística y Geografía (INEGI) México presentó una población de 119,938,473 habitantes en 2015, cuya mayor concentración se situó en el Estado de México con 16,187,608 habitantes, además la Ciudad de México presentó una población de 8,918,653 habitantes (INEGI, 2015a). De esta manera es posible decir que una de las problemáticas sociales que aqueja en mayor medida a la población mexicana es la violencia que se vive dentro del país. Así, se observa que en el 2014, mediante la Encuesta Nacional de Victimización y Percepción sobre Seguridad Pública 2015 (ENVIPE), más de 22 millones de habitantes han sido víctima de un delito; en donde el $51.4 \%$ eran mujeres y el restante $48.6 \%$ eran hombres (INEGI, 2015b).

Mientras que para la Ciudad de México se calculaban 36,019 víctimas

Correspondencia: Dr. Leonel Romero Uribe . Facultad de Estudios Superiores Zaragoza, UNAM.

Email:psic.ss@zaragoza.unam.mx (por cada cien mil habitantes), para el Estado de México se calculaban alrededor de 45,139 en el transcurso del 2014 (INEGI, 2015b). Consecuentemente, en 2016 se ubicó a México como el segundo país más violento, aludiendo que la causa principal del diagnóstico de violencia en el país fueron las muertes causadas por el narcotráfico (AFP, 2017).

El propósito de la investigación fue contrastar las diferencias en las creencias actitudinales de hombres y mujeres de la Ciudad y el Estado de México hacia la violencia (a través de la construcción y aplicación de un instrumento de medición de creencias y actitudes) Para los objetivos de la investigación, se distinguió entre los conceptos Violencia y Agresión, así como los comportamientos involucrados dentro de ellos.

Para algunas personas no existe diferencia entre violencia y agresión por lo que en ocasiones se suele usar de manera indiscriminada. Por consiguiente, en el concepto de agresión se pueden encontrar diversidad de definiciones y características que distintos autores le han atribuido a través de los años. Por ejemplo, Ther (2005) indica que la agresión no puede existir en el ser humano dado su condición evolucionada como Homo Sapiens (sólo podría llamarse violencia), mientras que Castillo (2006) entiende la agresión como la acción que tiene la intención de hacerle daño a otra persona. Adicionalmente Chaux (2003) argumenta que la agresión puede ser física, verbal (cuando se quiere herir a través de 
las palabras) o relacional (cuando se busca hacerle daño a las relaciones que tiene la otra persona o al estatus social que tiene en su grupo).

Según Castillo (2006), la psicología no es suficiente para alcanzar una completa comprensión de la agresividad dada su categoría multifactorial, por tanto, se requiere la interacción con otras ciencias, es por eso que alude a plantear el panorama desde diversos enfoques. Por lo cual, la definición de agresión tomada para la presente investigación hace referencia a un comportamiento básico y primario en la actividad de los seres humanos (y seres vivos en general); además ésta puede ser intencional, o no, con el fin de hacer daño a otra persona (Carrasco y González, 2006; Castillo, 2006).

Aunado a ello, se han descrito modelos y teorías en los cuales la agresión se encuentra vinculada a ellos; Freud (como se citó en Chapi, 2012) postuló la teoría del doble instinto, en la cual se concibe al hombre como dotado de una cantidad de energía dirigida hacia la destructividad, y que debe ser inevitablemente expresada de una forma u otra. Por otro lado, Dollar y Miller en 1944 (como se citó en Chapi, 2012) plantean el modelo de frustración-agresión, el cual explica que la agresión es siempre resultado de la frustración, y la frustración conduce ineludiblemente a la agresión, sin embargo, investigaciones recientes no sustentan dicha teoría, ya que no siempre se reacciona agresivamente pues puede encontrarse enmascarada por el contexto ambiental. Mientras que Tedeschi y Felson (1994, como se citó en Andreu, 2009), en la teoría de la interacción social, interpretan la conducta agresiva como conductas que son influidas por en entorno social, de esta manera se podría explicar el fenómeno de la agresión instrumental como una alternativa ante la solución de un conflicto o de obtención de una meta.

De manera similar la conceptualización de la violencia sufre un grave problema, debido a que como tal no existe un concepto o definición universal para explicar dicho fenómeno, esto porque abarca diversos aspectos en la vida de los seres humanos. Asimismo, es vista desde distintas perspectivas teóricas, las cuales intentan presentar una definición acorde a las características y requerimientos específicos a explicar en su ámbito, lo anterior da pauta para intentar conceptualizar a la violencia, tomando en cuenta las variables relacionadas con el fenómeno que se pretende estudiar.

En el sentido de lo anterior, se propone definir la violencia como un estadio más avanzado de la agresividad, pretende el uso deliberado de la fuerza física o el poder, de manera directa o indirecta, ya sea en grado de amenaza o efectivo, contra uno mismo, otra persona, grupo o comunidad; que cause o tenga probabilidades de causar lesiones, muerte, daños psicológicos, trastornos del desarrollo o privaciones (OMS, 2019).

Según el Informe mundial sobre la violencia y la salud (2002), se recurre a un modelo ecológico, para tomar en cuenta factores biológicos, sociales, políticos, económicos y culturales que podrían intervenir para que se produzcan y reproduzcan conductas violentas:

Nivel Individual: Factores biológicos y personales del individuo para explicar la tendencia de convertirse en alguien violento o en víctima de actos violentos; características demográficas, antecedentes psiquiátricos, conductas agresivas o antecedentes de haber sufrido algún tipo de maltrato.

Nivel relacional: Se analizan las relaciones con familia, amigos, pareja, compañeros de trabajo, las cuales pueden influir en la presencia de comportamientos violentos; pertenecer a una familia disfuncional, falta de vínculos emocionales o haber sufrido maltrato infantil.

Nivel comunitario: Explora contextos en los que la persona establece sus relaciones sociales con el objetivo de identificar si estos ámbitos pudiesen influir en el riesgo de cometer actos violentos.

Nivel social: Estructura de la sociedad (cultura), así como las normas establecidas en cuanto los efectos de la producción, reproducción o inhibición de actos violento y las condiciones políticas, sociales, económicas, educativas y sanitarias.
Siguiendo con el marco referencial, el Informe Mundial de Violencia y Salud (2002), divide las categorías en las cuales se dan los comportamientos violentos, de acuerdo con quién es el victimario y a qué tipo de violencia fue sometida.

Violencia según el victimario: Depende del tipo de persona que intente provocar algún daño, estas pueden ir desde personas externas hasta uno mismo:

Violencia Interpersonal: Abarca todos los actos de índole violenta cometidos por un individuo o un pequeño grupo de individuos hacia una o varias víctimas (pueden ser físicos, psíquicos, sexuales, privaciones y abandonos).

Violencia autoinfligida: Actos o comportamientos violentos que un individuo perpetúa contra sí mismo, abarca desde pequeñas heridas hasta la muerte. Este tema está rodeado de prejuicios y tabúes, incluso es criticado por la religión o cultura, ello dificulta la detección y prevención de los mismos, así como su tratamiento.

Violencia colectiva: Es el uso intencional de la violencia por personas que se identifican a sí mismas como miembros de un grupo frente a otro grupo o conjunto de individuos, con el fin de lograr objetivos políticos, económicos o sociales.

Violencia según el tipo de daño causado: A continuación, se enuncia una clasificación de violencia acorde a la Ley General de Acceso de las Mujeres a una Vida Libre de Violencia (2015, como se cita en CONAPO, 2018):

Violencia física: Definida como el uso de la fuerza física para provocar daño no accidental, o con algún tipo de arma u objeto, que pueda provocar (o no) lesiones internas y/o externas. Suele dejar lesiones en el cuerpo que pueden ser permanentes, provocar alguna discapacidad o causar la muerte.

Violencia psicológica: Cualquier acto u omisión que dañe la estabilidad mental. Se manifiesta como un largo proceso en el cual la víctima no se da cuenta que el agresor vulnera sus derechos, ya que el maltrato suele ser sutil. Puede consistir en abandono, descuido reiterado, insultos, humillaciones, entre otras.

Violencia económica: Se manifiesta mediante restricciones encaminadas a controlar el ingreso de las percepciones económicas y limita la autonomía de la víctima.

Violencia sexual: Acto que degrade o dañe el cuerpo y/o la sexualidad de la víctima y que por tanto atenta contra su libertad, dignidad e integridad física. Ocurre a cualquier persona y los abusadores pueden ser personas cercanas o desconocidos.

Una vez expuestos los conceptos de agresión y violencia, se abre paso al tema de investigación, por lo que se procederá a examinar la definición y estructura de las creencias actitudinales. De este modo, primeramente, se habla de creencias como verdades subjetivas, consideradas convicciones, de algo que se considera cierto. Así. se ha realizado la adopción de dos concepciones; la epistemológica en la cual se menciona un razonamiento de la realidad dando como resultado una idea y la psicológica en donde la creencia es considerada una disposición. Por lo que las creencias son todas aquellas ideas con las que contamos, que, aunque no se piense en éstas, dan como resultado un comportamiento de acuerdo a ellas, como si existieran realmente (Díez, 2017). Asimismo, Villoro (1982 como se citó en Ortiz, 2007) apunta a que las creencias son un conjunto de respuestas coherentes determinadas por un objeto o situación aprendida, las cuales se adquieren por medio de la interacción con el mundo, así como las personas que se encuentran en el medio.

Por lo anterior, la forma en que las personas construyen sus creencias 
se describe a través la máquina de creencias de Alcock (1995, como se citó en Morales, Moya, Giviria y Cuadrado, 2007), ésta hace referencia a que el ser humano desarrolla distintos sistemas de creencias para mantenerse, sin importar la obtención de la verdad, ni estar guiado por los principios de la lógica. Por lo tanto, el desarrollo de creencias es un proceso secuencial empezando con la selección de información, su moldeamiento en función de intereses y preocupaciones, la combinación con otras informaciones acumuladas, dando como resultado la producción de una nueva creencia congruente con las que ya se poseían.

Seoane y Garzón (1996) clasificaron las creencias por el contenido de los sistemas, teniendo tres dimensiones significativas:

Política o de organización social: Creencias sobre la forma y el funcionamiento de la sociedad en la que se vive, tanto en manifestaciones reales como los ideales, teniendo en cuenta las formas de vida que el sistema político permite y estimula.

Cultural o de conocimiento básico: Creencias y actitudes relacionadas con la naturaleza, la ciencia, la historia o alguna otra concepción relacionada con el mundo, incluyendo la percepción del tiempo histórico.

Social o de relaciones personales: Creencias sobre la interacción con los demás, la forma de relación o el cuidado de los sentimientos, donde se incluye las relaciones primarias.

En este punto es evidente que las creencias van acompañadas de otras creencias, relacionándose con actitudes, valores, opiniones, sentimientos, así como con orientaciones. Por ello, se perciben dos principales directrices, una donde se toma a la actitud como conjunto de creencias, y otra en que la creencia es elemento cognitivo de las actitudes (Seoane y Garzón, 1996). Lo cual destaca que las creencias son parte fundamental, al igual que una complementación, de las actitudes, esto ayuda a entender y poder explicar por qué las personas que sostienen diferentes creencias pueden presentar las mismas actitudes, así como a la inversa (Morales, 2006).

Con lo anteriormente descrito, se hace necesario mencionar las actitudes. De esta manera, las actitudes son predisposiciones para actuar negativa o positivamente frente a los objetos; teniendo en cuenta que la actitud constituye un estado hipotético con el que se pretende explicar cierta consistencia en el comportamiento de las personas, cuyo carácter principal sería la evaluación hacia un determinado objeto (Baró, 1990). Así, para la psicología social Baron y Byrne (2005) mencionan que las actitudes hacen referencia a valoraciones de una persona con respecto a un aspecto de la realidad; estas evaluaciones son positivas o negativas, dependiendo del tema, idea, grupo social, etcétera. Sin embargo, las actitudes suelen presentar tanto evaluaciones positivas como negativas hacia un mismo objeto o situación; esta conjugación de valoraciones es llamada ambivalencia de la actitud.

Liberman y Chaiken (1991, como se citó en Saldívar, 2005) mencionan que la noción de ambivalencia se encuentra en relación con las actitudes que una persona manifiesta hacia un objeto determinado, porque implican valoraciones positivas y negativas simultáneas en el terreno afectivo y en el cognitivo; dicho conflicto se resuelve cuando uno de los aspectos (el positivo o el negativo) se pondera de algún modo y da forma a la expresión final de la actitud, sea ésta de tipo cognitivo o afectivo.

Asimismo, dentro del desarrollo de las actitudes se encuentran algunas características donde se representan tres componentes que influyen en la ejecución de las actitudes directamente. La influencia de los tres factores, cognitivo, conductual y afectivo, explican en gran parte la predicción y ejecución del comportamiento deseado o esperado (Arnau y Montané, 2010). Según esta afirmación, las ideas, los comportamientos y las emociones influyen directamente en la manera de actuar Para estos autores, las tres dimensiones están íntimamente relacionadas entre sí; de este modo, los contenidos cognitivos influyen a su vez y, dependen, de los componentes afectivos y de los elementos conductuales en forma de hábitos de comportamiento y de destrezas.

De igual forma, Barón y Byrne (2005) argumentan cuatro maneras en las cuáles la formación de actitudes tiene lugar:

Condicionamiento clásico (Pavlov): Dentro de este punto, la formación de actitudes es un aprendizaje basado en la asociación.

Condicionamiento instrumental (Skinner): Las actitudes son establecidas mediante comportamientos seguidos por resultados positivos o contraproducentes para la persona que ejecuta el comportamiento, estas actitudes están orientadas a aprender a mantener un punto de vista.

Aprendizaje observacional (Bandura): Las actitudes son establecidas cuando las personas observan diferentes formas de comportamientos en las acciones de las personas que le rodean (ya sea directa e indirectamente).

Comparación social (Festinger): Las personas tienden a comparar su comportamiento y/o punto de vista con las demás personas, para así evaluar y concluir si dicha comportamiento o punto de vista es correcto, o no, con base a la realidad social en la que se desarrolla.

Baron y Byrne (2005) igualmente se cuestionaron acerca de las funciones que traen consigo las actitudes y argumentaron tres funciones principales de éstas. La primera función es respecto a que las actitudes suelen operar como esquemas de la realidad social del individuo, cuya finalidad es matizar las percepciones y pensamientos de dicho individuo. Una segunda función está relacionada a la autoestima, referente al hecho de que los comportamientos derivados de actitudes ayudan al individuo a mantener sus sentimientos de confianza y, así, reforzar sus actitudes porque se piensa que éstas son correctas. Finalmente, las actitudes son de autodefensa, es decir, funcionan como un filtro de información no deseada o que vaya en contra de su manera de comportarse o de sus actitudes.

A todo esto, ¿cómo se le llama al hecho de que una persona actúe de una forma contradictoria respecto a su actitud previa? si la consistencia entre actitudes y comportamiento es lo normal, cómo es que en ocasiones estos dos aspectos no tienen congruencia entre sí y aun así las personas siguen desempeñándose día con día en sus actividades cotidianas. Entonces ¿cuáles son las consecuencias de lo anteriormente descrito? Ovejero (1993) argumenta que la inconsistencia entre las actitudes y el comportamiento está acompañada de un cierto tipo de incomodidad en la persona que experimenta dicha inconsistencia.

Para este tipo de situaciones Saldivar (2005) hace mención del constructo propuesto por Festinger en 1957 llamado disonancia cognitiva, la cual se traduce como un "malestar" derivado de diferentes cogniciones contradictorias entre sí, de las cuales la persona que las experimenta debe elegir una sobre otra y actuar sobre su base. Sumando a lo anterior, Rosero y Montalvo (2015) mencionan que este malestar es un malestar psicológico, además de que su presencia es ocasionada cuando los pensamientos (cogniciones en el caso anterior) son diferentes a las acciones llevadas a cabo por el individuo que las tiene.

Con ello, Baró (1990) argumenta que, aunque la actitud está definida como una relación de sentido, no tiene por qué ligarse a un solo comportamiento, sino que la persona puede necesitar actuar de diverso modo precisamente para mantener el vínculo valorativo con el objeto de su actitud. De esta manera las actitudes no pueden ser el determinante único de las acciones humanas. Determinando así, que el conjunto de actitudes de una persona constituye su estructura ideológica, en cuanto canalización y producto psicosocial de los intereses propios de su grupo social. 


\section{MÉTODO}

\section{Justificación}

El estudio sobre las actitudes y creencias hacia la violencia, en comparación de la agresión, es una necesidad del contexto sociocultural actual, tomando en cuenta la creciente ola de violencia suscitada desde las estadísticas presentadas en el 2010 por el Instituto Ciudadano de Estudios Sobre la Inseguridad y en el 2015 y 2017 por el INEGI. Señalando, a su vez, la diferencia existente entre la concepción de la violencia contrastada por el sexo, dado que existe un mayor índice de violencia hacia las mujeres. Cabe resaltar que tanto las creencias como las actitudes son elementos esenciales del desarrollo de comportamientos manifestados por las personas; así el distinguir las causas que los generan, además de los elementos justificadores de la violencia, permite comprender el constructo de agresión y violencia en el contexto social. Por tal motivo una de las zonas de mayor implicación para el estudio de estos constructos es la zona oriente, siendo esta un lugar en donde se presentan un mayor índice de actos delictivos, estando en el área limítrofe de la Ciudad de México y el Estado de México.

\section{Pregunta de investigación}

¿Existen diferencias entre las creencias actitudinales de hombres y mujeres de la Ciudad y el Estado de México hacia la violencia?

\section{Objetivos}

Medir las creencias actitudinales de hombres y mujeres hacia la violencia.

Contrastar las diferencias en las creencias actitudinales de hombres y mujeres de la Ciudad y el Estado de México hacia la violencia.

\section{Hipótesis}

Existen diferencias entre las creencias actitudinales de hombres y mujeres de la Ciudad y el Estado de México hacia la violencia.

Existe mayor aceptación en las creencias actitudinales de los hombres de la Ciudad y el Estado de México hacia la violencia.

Existe mayor aceptación en las creencias actitudinales de las mujeres de la Ciudad y el Estado de México hacia la violencia.

\section{Definición operacional y conceptual de variables}

Variable dependiente: Creencias actitudinales. De acuerdo con lo que menciona Milton Rokeach (1968, como se citó en Aigneren, 2016) una actitud es una organización relativamente duradera de creencias en torno a un objeto o una situación, las cuales predisponen a reaccionar preferentemente de una manera determinada.

Variable independiente: Sexo (hombre y mujer).

\section{Tipo de estudio}

El tipo de estudio descriptivo, ya que se describe el fenómeno observado tal y como se manifiesta en el contexto social. Así mismo de corte transversal debido a que se tomó una sola medición en un momento específico y no hubo seguimiento (Ato y Vallejo, 2015).

\section{Enfoque}

El enfoque utilizado en la presente investigación fue cuantitativo, debido a que de acuerdo a lo que menciona Sampieri (2014), se tendió a predecir el comportamiento de las variables estudiadas, así como encontrar una relación estadísticamente significativa entre las mismas.

\section{Tipo de diseño}

Se hizo uso del diseño retrospectivo o Ex post facto dado que el fenó- meno tiene lugar antes del comienzo del estudio, por tal motivo la recogida de datos se llevó a cabo con información ya existente de diferentes fuentes (Ato y Vallejo, 2015).

\section{Muestra}

La muestra constó de 294 habitantes, mayores de edad, provenientes de la Ciudad de México y el Estado de México.

\section{Muestreo}

El tipo de muestreo fue no probabilístico, a través del muestreo accidental por cuota, puesto que la muestra se conformó acorde a las características propuestas anteriormente (Otzen y Manterola, 2017; Sampieri, 2014).

\section{Instrumento}

Se construyó un cuestionario tipo escala Likert para medir las creencias actitudinales hacia la violencia. Dicho instrumento constó de 55 reactivos, los cuales se clasifican de acuerdo con cinco dimensiones de medición de la violencia que son: Interpersonal física, Interpersonal psicológica, Autoinfligida, Colectiva física y Colectiva psicológica. Los cuales a su vez se subdividen en doce componentes; violencia justificada de género (5 reactivos), violencia en la pareja (3 reactivos), violencia inaceptable (3 reactivos), violencia sexual (3 reactivos), violencia familiar (4 reactivos), coerción sexual (3 reactivos), autocastigo (4 reactivos), conductas de riesgo (3 reactivos), violencia a grupos vulnerables (5 reactivos), discriminación ( 5 reactivos), prejuicios (4 reactivos) y estereotipos (3 reactivos).

\section{Análisis de resultados}

El programa estadístico SPSS se empleó para analizar los resultados obtenidos mediante un análisis factorial con el fin de identificar el grado de explicación y confiabilidad que obtiene cada subescala respecto al instrumento en su totalidad, además de agrupar los reactivos en componentes específicos para una medición detallada. También, se aplicó una serie de ANOVAS de un factor para determinar si existen diferencias significativas entre la población. Así como la aplicación de una correlación de Pearson para observar el nivel de relación entre los distintos tipos de violencia.

\section{RESULTADOS}

Los datos se analizaron con el paquete estadístico IBM-SPSS versión 24 , en el que primeramente se obtuvieron estadísticos descriptivos de la muestra bajo estudio. De esta manera, se observa que la muestra estuvo integrada por $49 \%$ de mujeres (144 participantes) y $51 \%$ de hombres (150 participantes), de los cuales el $92 \%$ está soltero y únicamente el $8 \%$ se encontraba casada al momento de la aplicación del instrumento. Asimismo, en la figura 1 se observa que la distribución entre los distintos niveles académicos fue alrededor cuatro categorías; sin embargo, se percibe una acumulación en el nivel Profesional, con el 63\% de los participantes.

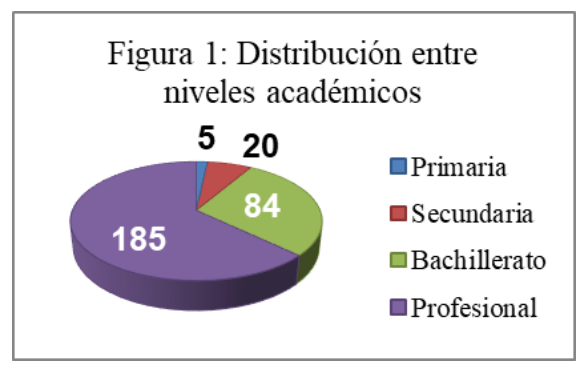

Los datos representan el número de personas que conformaron cada categoría 
La muestra provenía de diferentes alcaldías que conforman la Ciudad de México, así como de distintos municipios del Estado de México; no así la distribución de la muestra fue uniforme, dado que las zonas que presentaron mayor porcentaje fueron Iztapalapa (aproximadamente con el $20 \%$ ), Nezahualcóyotl (con el 14\%), Iztacalco (con el 11\%), Ecatepec (con el 8\%) e Ixtapaluca (con el 7\%). Así, el restante $40 \%$ de los individuos provinieron de 26 diferentes zonas.

En cuanto al instrumento propuesto para medir las actitudes, se realizó un análisis factorial. Así, en la subescala violencia física se obtuvo una varianza total que explica el $48 \%$, además dentro de ésta sólo se agruparon 16 reactivos en cuatro componentes. Los componentes derivados de la subescala son violencia justificada de género (cuya $\alpha$ es igual a .680), violencia en la pareja ( $\operatorname{con} \alpha=.534)$, violencia inaceptable (con $\alpha$ $=.328)$ y violencia sexual $(\operatorname{con} \alpha=-.139)$.

Para la subescala de violencia psicológica, los resultados presentaron una varianza total que explicaba el $42 \%$ de la subescala, al mismo tiempo de estar agrupados en los componentes violencia familiar (con $\alpha$ $=.572)$ y coerción sexual $(\operatorname{con} \alpha=.409)$. En el tercer factor, violencia autoinfligida, los reactivos se agruparon en dos componentes distintos; autocastigo (con $\alpha=.614$ ) y conductas de riesgo ( $\operatorname{con} \alpha=.012$ ). Para así ofrecer una explicación del $42.326 \%$ de esta subescala.

El análisis de la cuarta subescala, violencia colectiva física, dentro de esta subescala los reactivos se encuentran agrupados en el componente violencia a grupos vulnerables ( $\operatorname{con} \alpha=.485$ ); este componente explica el $22.285 \%$ de la varianza total de esta subescala. Finalmente, para la subescala de violencia colectiva psicológica se observa que los reactivos se agrupan en tres componentes; discriminación (con $\alpha=.692$ ), prejui$\operatorname{cios}(\operatorname{con} \alpha=.454)$ y estereotipos (con $\alpha=.489$ ). A su vez, éstos reportan una varianza total explicada del $42.458 \%$ para esta subescala.

Por otra parte, se realizó una serie de ANOVAS para cada componente, contrastando con datos sociodemográficos provenientes de la muestra. Así, el análisis entre los componentes y el sexo; los resultados arrojaron un nivel crítico (sig.) estadísticamente significativo en violencia justificada de género $(\mathrm{p}=0.016)$, violencia sexual $(\mathrm{p}=0.003)$, y discriminación $(\mathrm{p}=0.001)$. Por lo cual, se apunta que hay diferencias significativas entre hombres y mujeres en los componentes antes mencionados.

Después, los resultados del análisis entre componentes y el estado civil de los integrantes muestra un nivel estadísticamente significativo en la subescala violencia sexual $(p=0.032)$ y estereotipos $(p=0.001)$. Contrariamente, los resultados entre los componentes y el nivel académico se observó un nivel estadísticamente significativo en la subescala violencia justificada de género $(\mathrm{p}=0.009)$, autocastigo $(\mathrm{p}=0.021)$, violencia a grupos vulnerables $(\mathrm{p}=0.011)$, discriminación $(\mathrm{p}=0.011)$ y estereotipos $(\mathrm{p}=0.008)$.

Entre las subescalas agrupadas y rangos de edad los resultados arrojaron un nivel estadísticamente significativo en violencia a en la pareja $(\mathrm{p}=0.018)$ y estereotipos $(\mathrm{p}=0.001)$. Culminando el análisis con los resultados entre los componentes y zonas de residencia, se percibió un nivel estadísticamente significativo únicamente en violencia en la pareja $(\mathrm{p}=0.032)$. Por lo cual, se apunta que hay diferencias significativas entre los residentes de la Ciudad de México y residentes del Estado de México.

Posteriormente se realizó una Correlación de Pearson entre los 12 diferentes componentes de la escala, los resultados se muestran en la tabla 1. En el primer factor, Violencia justificada de género, se presentan correlaciones moderadas significantes en el nivel de $\mathrm{p}<0.01$ (bilateral) con los factores; violencia a grupos vulnerables $(p=.606)$, discriminación $(\mathrm{p}=.560)$, violencia familiar $(\mathrm{p}=.518)$, autocastigo $(\mathrm{p}=.508)$, violencia inaceptable $(\mathrm{p}=.455)$ y estereotipos $(\mathrm{p}=.413)$. Por otra parte, el factor de Violencia en la pareja presento únicamente una correlación moderada significante en el nivel de $\mathrm{p}<0.01$ (bilateral) con el factor de coerción sexual $(\mathrm{p}=.585)$.

Contrariamente, el factor Violencia familiar presenta correlaciones significativas moderadas en el nivel $\mathrm{p}<0.01$ (bilateral) con los factores violencia a grupos vulnerables $(\mathrm{p}=.434)$ y discriminación $(\mathrm{p}=.439)$. Para el factor Autocastigo presenta correlaciones moderadas significante en el nivel de $\mathrm{p}<0.01$ (bilateral) con el factor de violencia a grupos vulnerables $(\mathrm{p}=.512)$ y prejuicios $(\mathrm{p}=.447)$, mientras que presenta una correlación de $\mathrm{p}=.481$ para el factor de estereotipos en el nivel de $\mathrm{p}<0.05$.

Por último, en el factor Violencia a grupos vulnerables se manifiestan correlaciones moderadas significantes en el nivel de $\mathrm{p}<0.01$ con una significancia de .468 para el factor de discriminación y de .448 para el factor de estereotipos. Cabe resaltar que las correlaciones entre los componentes restantes tienen una significancia $\mathrm{p}<0.400$, o manifiestan correlaciones no significativas.

\section{Tabla 1:}

Correlación de Pearson entre factores

\begin{tabular}{|c|c|c|c|c|c|}
\hline & & CORREL & ACIONES & & \\
\hline & $\begin{array}{l}\text { Violencia } \\
\text { justificada } \\
\text { de género }\end{array}$ & $\begin{array}{c}\text { Violencia } \\
\text { en la } \\
\text { pareja }\end{array}$ & $\begin{array}{l}\text { Violencia } \\
\text { familiar }\end{array}$ & Autocastigo & $\begin{array}{c}\text { Violencia a } \\
\text { grupos } \\
\text { vulnerables }\end{array}$ \\
\hline $\begin{array}{l}\text { Violencia } \\
\text { inaceptable }\end{array}$ & $.455 * *$ & & & & \\
\hline $\begin{array}{l}\text { Violencia } \\
\text { familiar }\end{array}$ & $.518^{* *}$ & & & & \\
\hline $\begin{array}{l}\text { Cohesión } \\
\text { sexual }\end{array}$ & & $.585^{* *}$ & & & \\
\hline Autocastigo & $.508^{* *}$ & & . & & \\
\hline $\begin{array}{l}\text { Violencia a } \\
\text { grupos } \\
\text { vulnerables }\end{array}$ & $.606^{* *}$ & & $.434^{* *}$ & $.512 * *$ & \\
\hline Discriminación & $.560 * *$ & & $.439 * 8$ & $.481^{*}$ & $.468 * *$ \\
\hline Prejuicios & & & & $.447^{* *}$ & \\
\hline Estereotipos & $.413^{* 8}$ & & . & & $.448 * *$ \\
\hline
\end{tabular}

Datos marcados con $* *$ señalan una correlación significativa en el nivel 0.01 (bilateral), datos marcados con * señalan una correlación significativa en el nivel 0.05 (bilateral)

\section{DISCUSIÓN}

Como se menciona al inicio de la investigación, uno de los principales problemas que aqueja a México es la violencia que se vive al interior del país. ¡Resaltando el hecho de que en 2014 se calcularon más de 22 millones de habitantes que han sido víctima de un delito; en donde el $51.4 \%$ eran mujeres y el restante $48.6 \%$ eran hombres (INEGI, 2015b).

De esta manera y gracias al análisis de resultados se puede observar que en la primera subescala del instrumento, Violencia Física, se observan cuatro componentes; de los cuales el componente violencia justificada de género muestra un alfa aceptable mayor a 0.6 , mientras que violencia en la pareja muestra un alfa moderada de 0.534 . Lo anterior puede aludir a que los reactivos dentro de ambos componentes se mueven alrededor de diferentes tipos de violencia, es decir, además de estar dentro del factor de violencia física, también se encuentra dentro del tipo de violencia relacional (por las relaciones con terceras personas, que pueden influir en la presencia de comportamientos violentos) y dentro de la violencia social (con vínculo a la estructura de la sociedad, es decir la cultura en la que se desarrollan los individuos) acorde a lo descrito por la CONAPO (2018).

Caso contrario es lo que se presenta con los componentes violencia inaceptable y violencia sexual, en las cuales se observa un alfa de .328 y de -.139 respectivamente; este último componente se asocia con la definición de violencia sexual (cualquier acto que degrade o dañe el cuerpo y/o la sexualidad de la víctima y que por tanto atenta contra su libertad, dignidad e integridad física) dada por la CONAPO (2018). Estos resultados pueden deberse a que además de que los componentes se mueven sobre distintos tipos de violencia, los reactivos están enunciados acorde a lo 
socialmente aceptable y pueden generar deseabilidad social en aquel que responde el instrumento. Esto puede asociarse a lo dicho por Baron y Byrne (2005) debido a que existen aspectos específicos en los cuales las personas optan por mostrar una actitud diferente a la que se posee, ya sea por la situación en la que se encuentran o por disminuir la presión social; originándose así una disonancia cognitiva. Así, dentro de esta subescala los primeros dos componentes explican la mayor parte de este factor. Sin embargo, un aspecto importante a destacar es que la varianza total explicada es menor del 50\% (siendo 48.193).

Para la segunda subescala, Violencia Psicológica, se observan dos componentes; estos son violencia familiar y coerción sexual, cuya $\alpha$ es de .572 y .409 (con pesos factoriales mayores a .50 en su mayoría). Con ello, esta subescala muestra un mayor acoplamiento en cuanto a la variable a explicar, esto porque solamente fue descartado un reactivo. Por tanto, el componente de violencia familiar se relaciona con la definición propuesta por La Ley contra la Violencia Intrafamiliar (citada en PNUD, 2013), esto hace referencia a cualquier acción u omisión (directa o indirecta) que cause daño, sufrimiento físico, sexual, psicológico o la muerte a las personas integrantes de una familia, con una confiabilidad moderada.

Con base en las dos primeras subescalas, se percibe que se relacionan mayormente con la Violencia Interpersonal; la cual se refiere a los actos de índole violenta cometidos por un individuo o un pequeño grupo de individuos hacia una o varias víctimas (pueden ser físicos, psíquicos, sexuales, privaciones y abandonos) dentro de este se encuentra la violencia familiar, de pareja, en la escuela, el trabajo, violencia juvenil, entre otras (Informe mundial sobre la violencia y la salud, 2002).

Dentro de la tercera subescala, Violencia Autoinfligida, se apunta un mayor acercamiento con la definición citada en la introducción, ésta establece que la violencia autoinfligida hace referencia a los actos o comportamientos violentos que un individuo perpetuo contra sí mismo (Informe mundial sobre la violencia y la salud, 2002). Dentro de la subescala se agruparon los reactivos en dos componentes, Autocastigo y Conductas de riesgo; estos a su vez explican el $42 \%$ de la variable.

La cuarta subescala, Violencia Colectiva Física, está explicada en un 22\% por el componente violencia a grupos vulnerables con una confiabilidad moderada del .485; por lo tanto, esta relación debe tomarse con reserva. Esto podría deberse a que, en su mayoría, los reactivos no logran contemplar la gran diversidad de grupos vulnerables existentes, además de centrarse en aspectos muy específicos y particulares de cada grupo representado, obviando las diferentes dificultades que enfrentan estos grupos. No obstante, la última subescala, Violencia Colectiva Psicológica, muestra una agrupación de reactivos en tres diferentes componentes, cuya explicación de la variable es de poco más del $44 \%$, debido a que un componente se descarta por no cumplir con las normas estadísticas. De esta manera, los tres componentes (Discriminación con $\alpha=.692$, Prejuicios con $\alpha=.454$ y Estereotipos con $\alpha=.489$ ) se pueden aludir a otros tipos de violencia; como los son violencia relacional y violencia social (descritas anteriormente), así como a la violencia estructural (definida como aquellos aspectos ideológicos y representacionales que justifican o engrandecen la violencia de alguna manera).

Estas dos últimas subescalas explican (con reservas) la violencia colectiva, definida como aquella violencia por personas que se identifican a sí mismas como miembros de un grupo frente a otro grupo o conjunto de individuos, con el fin de lograr objetivos políticos, económicos o sociales (Informe mundial sobre la violencia y la salud, 2002).

Con base en lo anterior, el panorama general describe como cada subescala está integrada por diferentes componentes que explican en todos los casos menos del $50 \%$ de cada variable, con lo cual se tiene que mencionar que los resultados deben tomarse con cautela. Puesto que de los doce componentes finales que integran el instrumento, únicamente tres de ellos cumplen con una confiabilidad mayor al .60, esto puede corresponder a que los reactivos mantienen relación con diferentes tipos de violencia.

Por otra parte, se realizaron una serie de ANOVAS contra las variables sociodemográficas de la población estudiada y se obtuvieron diferencias significativas. Primeramente, se encontró diferencias significativas entre el sexo con respecto a la discriminación, la violencia justificada de género y violencia sexual; estos dos últimas pueden deberse al énfasis que se ha puesto en la violencia en contra de la mujer dado que son mayormente visibles y juzgadas a la luz de la sociedad y de la ley a diferencia de años anteriores. Además de que se adscriben como factores facilitadores de comportamientos violentos, estos comportamientos guardan relación con el establecimiento de creencias y con la manifestación de actitudes; destacándose el hecho de que la disonancia cognitiva puede presentarse porque el tema de violencia de género suele originar deseabilidad social. Por lo anterior, estos resultados apuntan a que existen diferencias significativas en las creencias actitudinales entre hombres y mujeres de la Ciudad y el Estado de México con respecto a la violencia, con ello se acepta la hipótesis planteada al inicio de la investigación. Sin embargo, estas diferencias sólo son significativas en los tres componentes ya mencionados.

Asimismo, el estado civil muestra diferencias significativas entre solteros y casados en cuanto a la violencia sexual y estereotipos; sin embargo, cabe resaltar el hecho de que más del $90 \%$ de los participantes estaba soltero al momento de la aplicación, por lo cual las diferencias deben tomarse con cautela. Aunado a ello, se observan diferencias en cuanto al nivel académico dentro de cinco componentes; pero, al igual que el caso anterior, se observa una acumulación de 185 participantes de nivel profesional (más del 60\%), lo cual puede entorpecer el análisis para identificar diferencias estadísticamente significativas. Sumándose la cuestión de que en los dos primeros niveles (primaria y secundaria) manifiestan un acumulado menor del 8 por ciento.

Concerniente a la localidad, los resultados muestran solamente en el componente de violencia en la pareja una diferencia significativa entre personas provenientes de la Ciudad México y las personas provenientes del Estado de México. Esto se podría aludir a que la Ciudad presenta mayor número de violencia a diferencia del Edo. México, además de que según la AFP (2017) México se situó como el segundo país más violento del mundo en 2017. Por lo que las personas al convivir en ambientes violentos y de constantes agresiones e inseguridad, tienen mayor facilidad de compartir normas subjetivas de actitudes con vínculo a una ideología de los comportamientos, pensamientos, actitudes y creencias socialmente aceptados; y con ello, ser más propensos a cometer actos violentos en contra de su pareja, puesto a que el contexto, la cultura y el ambiente así lo facilitan (cabe aclarar que hay factores que pueden disminuir dichos comportamientos).

A manera de conclusión, se menciona que gracias a la investigación se pudo observar como las diferentes clasificaciones de distintos autores guardan relación con aspectos particulares de cada tipo de violencia; que, si bien hay una diversidad en cuanto a tipos, la violencia se interrelacionan y se construye como un constructo amplio que guarda un vínculo con las vivencias cotidianas de la sociedad mexicana. Esto debido a que México hoy en día enfrenta una crisis de inseguridad, con lo cual esta escala nos permite un acercamiento a las creencias actitudinales mantenidas entre un sector de la población mexicana. Además de observarse como las diferencias entre zonas relativamente cercanas sólo se manifiesta en puntuales factores; por ende, se puede abordar la problemática de la violencia desde diferentes perspectivas, pero de un modo mayormente homogéneo en cuanto a zonas cercanas, dado que éstas presentan similitudes en cuanto a las actitudes de sus pobladores.

Por todo ello y como se ha venido gestando desde investigaciones atrás, este estudio ayuda a percibir como se pueden dar las relaciones entre diferentes tipos de violencia y las semejanzas que hay entre creencias actitudinales; que, contando con una muestra equitativa, pueden obtenerse resultados que permitan a los psicólogos encargados de elaborar planes de acción centrados en la disminución de la violencia tanto a nivel general como familiar, sexual de género, autoinfligida, entre otras.

\section{REFERENCIAS}

AFP. (2017). El narcotráfico provocó en 201623 mil asesinatos en México: IISS. La jornada. Recuperado de https:// www.jornada.com.mx/2017/05/10/politica/003n1pol

Aigneren, M. (2016). Técnicas de medición por medio de escalas. Universidad de Antioquia, Facultad de Ciencias Sociales y Humanas. Centro de estudios de opinión, pp 1-25.

Andreu, J. (2009). Propuesta de un modelo integrador de la agresividad impulsiva y premeditada en función de sus bases motivacionales y socio-cognitivas. Psicopatología clínica, legal y forense, 9 (1). Recuperado de https://masterforense.com/ pdf/2009/2009art5.pdf 
Arnau, S. L. y Montané, C. J. (2010). Aportaciones sobre la relación conceptual entre actitud y competencia, desde la teoría de cambio de actitudes. Electronic Journal of Research in Educational Psychology, 8 (3), pp 1283-1302.

Ato, M. y Vallejo, G. (2015). Diseños de investigación en Psicología. España: Pirámide.

Baró, I. M. (1990). Las actitudes: su concepto y valor. En Acción e Ideología: Psicología Social Desde Centroamérica. (pp. 241355). El salvador: UCA Editores.

Barón, R. Y Byrne, D. (2005). Psicología social. España: Pearson Prentice hall

Carrasco, M. y González, M. (2006). Aspectos conceptuales de la agresión: definición y modelos explicativos. Acción Psicológica, 4(2). Recuperado de http://revistas.uned.es/index.php/ accionpsicologica/article/view/478

Castillo, M. (2006). Comportamiento agresivo y sus diferentes enfoques. Psicogente, 9 (15), pp 166-170.

Chapi, J. (2012). Una revisión psicológica las teorías de la agresividad. Revista Electrónica de Psicología Iztacala, 15 (1), pp 80-93.

Chaux, E. (2003). Agresión reactiva, agresión instrumental y el ciclo de la violencia. Revista de Estudios Sociales, 15 (1), pp 47 58

CONAPO. (2018). Prevención de la Violencia en la Familia: ¿Qué onda con..? Los tipos de violencia. Recuperado de https:// www.gob.mx/cms/uploads/attachment/file/303594/ Prevencion_de la_violencia_Tipos_de_Violencia.pdf

Díez, A. (2017). Más sobre la interpretación (II). Ideas y creencias Revista de la Asociación Española de Neuropsiquiatría. 37 (131) pp 127-143.

INEGI. (2015a). Encuesta Intercensal. Recuperado de

https://www.inegi.org.mx/contenidos/programas/ intercensal/2015/doc/eic 2015 presentacion.pdf

INEGI. (2015b). Encuesta Nacional de Victimización y Percepción sobre Seguridad Pública 2015. Recuperado de

https://www.inegi.org.mx/contenidos/programas/envipe/2015/ doc/envipe 2015 presentacion nacional.pdf

Informe mundial sobre la violencia y la salud. (2002). Organización Panamericana de la Salud, Oficina Regional para las Américas de la Organización Mundial de la Salud. Recuperado de

https://www.who.int/violence injury prevention/violence/

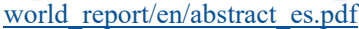

Morales. J.F. (2006). Psicología social. México: McGraw Hill.

Morales, J., Moya, M., Gaviria, E. y Cuadrado, I. (2007). Psicología social. España: McGraw Hill.

Organización Mundial de la Salud (OMS, 2019). Violencia. Recuperado de https://www.who.int/topics/violence/es/

Ortiz, G. (2007). Una definición muy general. Un comentario a la definición de creencia de Villoro. Signos filosóficos, 4 (18) pp. 181-189.

Otzen, T. y Manterola, C. (2017). Técnicas de Muestreo sobre una Población a Estudio. International Journal of Morphology, 35 (1), pp. 227-232. Recuperado de https://scielo.conicyt.cl/ pdf/ijmorphol/v35n1/art37.pdf

Ovejero, A. (1993). La teoría de la disonancia cognitiva. Psicothema, 5 (1). pp 201-206.

PNUD. (2013). Informe Regional de Desarrollo Humano 2013-2014. Seguridad ciudadana con rostro humano: diagnóstico y propuestas para América Latina. Recuperado de https:// www.undp.org/content/dam/rblac/img/IDH/IDH-AL \% 20Informe $\% 20$ completo.pdf
Rosero, M. y Montalvo, H. (2015). La disonancia cognitiva como factor motivador en las decisiones del consumidor: caso smartphones. Revista de Ciencias de la Administración y de la Economía, 5 (10).

Saldívar, A. (2005). El conflicto desde una perspectiva de género: elementos para el análisis de la dinámica de las relaciones entre mujeres y hombres. Revista de Ciencias Sociales y Humanidades (59).

Sampieri, R. H. (2014). Metodología de la Investigación. México: Interamericana Editores.

Seoane, J. Y Garzón, A. (1996). El marco de investigación del sistema de creencias postmodernas. Psicología política. 13, 81 - 98.

Ther, F. (2005). Entre la agresión y la violencia: ¿Aspectos constitutivos del comportamiento humano o de la constitución del Hominilupus cultural?. Textos antropológicos, 5 (1). Recuperado de https:// www.redalyc.org/pdf/447/44740216.pdf 